and 1 ) as $1621 \cdot 3,3019 \cdot 0,1343 \cdot 9,3069 \cdot 0,950 \cdot 0$ and 1100. Of these six, the last one has not yet been observed, but 3069.0 and 950.0 have been recorded by Bonner in the liquid. That they do not appear in the gas is presumably due to the very low intensity which may be expected of them. The first three, namely $1621 \cdot 3,3019 \cdot 0$ and $1343 \cdot 9$, need special mention, as they should be intense and exhibit a high degree of polarization in the Raman spectra.

I have made a careful study of the state of polarization of two of these three intense Raman lines. Details will be given elsewhere and only the results mentioned here. Depolarization factors of $0 \cdot 10$ and 0.17 respectively have been obtained for lines $3019 \cdot 0$ and $1343 \cdot 9$. The depolarization factor for $162 \mathrm{I} \cdot 3$ could not be determined with as good an accuracy, but the results show it to be also of the same low order. Such low depolarization factors are in complete accord with the theory.

The fact that two weak lines at 2880 and 1656 which are not fundamentals have been recorded in the Raman spectrum both by Bonner and by $m e$ in the liquid and the gas respectively is interesting. As has been suggested by Bonner, 1656 may be regarded as the overtone of an inactive fundamental at 825 , appearing as a result of its proximity to 1620 (similar instances occur in the Raman spectra of carbon dioxide, carbon disulphide, etc.), but the origin of 2880 is not at all clear.

Department of Physics, Andhra University, Waltair, India. Oct. 24.

\footnotetext{
${ }^{1}$ Bonner, J. Amer. Chem. Soc., 58, 34 (1936).
}

'Dickinson, Dillon and Rassetti, Phys. Rev., 34, 582 (1929).

'Sutherland and Dennison, Proc. Roy. Soc., A, 148, 250 (1935).

- Delfosse, Ann. Soc. Sci. Bruxelles, B, 45, 114 (1935).

${ }^{5}$ Manneback and Verleysen, NATURE, 138, 367 (1936).

\section{Dissociation Energy of Carbon Monoxide and the Abundance of Elements in Stellar Atmospheres}

A GREAT deal of work has been carried out by many investigators during the last two years in order to determine the heat of dissociation of carbon monoxide by means of spectroscopic methods. But as yet this very important figure is not known with any certainty, the values proposed by different authors varying between 11.06 and 6.9 e.v.; however, only the values $9 \cdot 1$ e.v. and less are not in contradiction with existing spectroscopic and thermochemical data.

The purpose of the present letter is to direct attention to the fact, that from all the proposed values for $D(\mathrm{CO})$ equal or less than $9 \cdot 1$ e.v., only the value $D(\mathrm{CO})=9 \cdot 1$ e.v. proposed by Goldfinger, Lasareff and Rosen ${ }^{1}$ and by Herzberg ${ }^{2}$ is in good agreement with our actual theoretical and observational knowledge of the composition of the atmospheres of late-type stars. A detailed investigation of this question has proved that : either the heat of dissociation of $\mathrm{CO}$ is really $D(\mathrm{CO})=9 \cdot 1 \mathrm{e.v}$., and the related value of the heat of dissociation of $\mathrm{CN}$ is $D(\mathrm{CN})=5.97$ e.v., or the abundances of light atoms in the main sequence as postulated by Cambresier and Rosenfeld ${ }^{3}$ and by Russell ${ }^{4}$ must be entirely revised.
Owing to the important role played by $D(\mathrm{CO})$ in all the calculations of molecular abundances in the late-type stars, the astrophysical evidence for the proposed value of $D(\mathrm{CO})$ seems worth mentioning.

Details on the subject will be published elsewhere.

M. Nicolet.

Department of Astrophysics, University, Liège.

1 NATURE, 135, 1077 (1935); 136, 226 (1935); C.R., 201, 958 (1935). 2 NATURE, 137, 620 (1936).

Mon. Not. Roy. Ast. Soc., 98, 711 (1933).

- Astrophys. J., 79, 281 (1934).

\section{Hydrogenation of Vitamin $B_{1}$}

WHEN treated with platinum-black or with hydro. sulphite, vitamin $B_{1}$ is easily reduced. Crystalline preparations from Hoffmann-La Roche and from I. G. Farben were used. They gave identical results. Hydrogenation with platinum-black was measured in Warburg-Barcroft manometers. In phosphate solution at $p \mathrm{H} 7.5,0.94 \mathrm{~mol} \mathrm{H}_{2}$ was absorbed per mol vitamin (average of three experiments).

To measure the reduction by hydrosulphite, the manometric method described by $O$. Warburg, Christian and Griese $\theta^{1}$ has been used. With this method, in bicarbonate solution, the acid formation due to oxidation of hydrosulphite $\left(\mathrm{Na}_{2} \mathrm{~S}_{2} \mathrm{O}_{2}\right)$ to acid sulphite is measured. One mol of acid is equivalent to one atom of hydrogen. But in many cases $^{2}$, in addition to that, acid is derived from the reduction process itself.

It has been found that $2 \cdot 76 \mathrm{mols}$ of acid are produced in the reaction between hydrosulphite and vitamin (average of four experiments). The reaction is finished after three hours at $25^{\circ} \mathrm{C}$. No acid formation takes place when the vitamin is treated with sulphite at $p H \quad 7 \cdot 8$. This possibility had to be excluded on account of the reaction between $\mathrm{NaHSO}_{3}$ and vitamin at $p H 5^{3}$. Then, of the $2 \cdot 76 \mathrm{mols}$ of acid, only two can be accounted for by oxidation of hydrosulphite, because only two atoms of hydrogen are added by platinum-black. The additional acid must be derived from the reduction process itself. Therefore it is assumed that the reduction takes place at the double bond closest to the quaternary nitrogen ${ }^{3}$. Then only one hydrogen is added; the other one is split into an electron and a hydrogen ion. The electron neutralizes the nitrogen and acid is formed. Tentatively the reduction of vitamin $B_{1}$ can be formulated as follows:<smiles></smiles><smiles>CC1=C(CO)SC[NH+]1Cc1c[nH]nc1C</smiles> 